\title{
Triplet chemotherapy combination with cisplatin, gemcitabine and docetaxel in patients with chemotherapy-naive advanced non-small cell lung cancer
}

\author{
FARUK TAS, FATMA SEN, NESE GUNEY, SERKAN KESKIN and HAKAN CAMLICA \\ Department of Medical Oncology, Institute of Oncology, Istanbul University, Istanbul 34390, Turkey \\ Received November 19, 2012; Accepted February 14, 2013
}

DOI: $10.3892 / \mathrm{ol} .2013 .1205$

\begin{abstract}
The synergistic effects of new generation chemotherapeutics when combined with cisplatin have encouraged the development of new triplet combination regimens in the treatment of advanced non-small cell lung cancer (NSCLC). The aim of this study was to evaluate the feasibility of triplet chemotherapy using weekly cisplatin-gemcitabine-docetaxel (CGD) for patients with chemotherapy-naive NSCLC. Twenty-seven patients with stage IIIB/IV disease and performance status of 0 to 2 were included in this prospective trial. A combination of gemcitabine $750 \mathrm{mg} / \mathrm{m}^{2}$, cisplatin $25 \mathrm{mg} / \mathrm{m}^{2}$ and docetaxel $25 \mathrm{mg} / \mathrm{m}^{2}$ was administered on days 1,8 and 15 , with cycles repeated every 3 weeks. Leucopenia and/or neutropenia and to a lesser extent thrombocytopenia were the main dose-limiting toxicities. Grade III-IV neutropenia and thrombocytopenia occurred in 26 and $7 \%$ of the patients, respectively. Only one patient developed febrile neutropenia. Dose reductions were required in $26 \%$ of patients, delays in $44 \%$ of patients and early treatment discontinuation in $15 \%$ of patients. The overall response rate was $52 \%$ and all of them experienced a partial response. The median progressionfree (PFS) and overall survival (OS) times were 6 and 13 months, respectively. The one-year survival rate was $46 \%$. In conclusion, weekly administration of CGD is an active firstline therapy with acceptable toxicity in advanced NSCLC patients.
\end{abstract}

\section{Introduction}

Non-small cell lung cancer (NSCLC) accounts for over $80 \%$ of patients with lung cancer, and nearly two-thirds of NSCLC patients present with advanced disease (stage IIIB/IV). Cisplatin-based chemotherapy with best supportive care (BSC)

Correspondence to: Dr Faruk Tas, Institute of Oncology, Istanbul University, Capa, Istanbul 34390, Turkey

E-mail: faruktas2002@yahoo.com

Key words: cisplatin, docetaxel, gemcitabine, non-small cell lung cancer, triplet chemotherapy, weekly has been demonstrated to have statistically significant survival advantage when compared with BSC alone in advanced NSCLC patients $(1,2)$. Cisplatin-based doublet chemotherapy with third generation cytotoxic agents, such as the taxanes vinorelbine or gemcitabine has further improved survival rates when compared with older regimens such as vindesine or etoposide $(3,4)$. Currently, doublet chemotherapy including a platinum and a third generation cytotoxic is accepted as a standard regimen for advanced NSCLC. Adding a third active drug is one of the further efforts to improve treatment outcome.

Gemcitabine, a pyrimidine nucleoside antimetabolite, is one of the most promising new cytotoxic agents. It has shown activity in a variety of solid tumors, but appears to be very active in the treatment of NSCLC (5). Gemcitabine in combination with cisplatin has synergistic effects without overlapping toxic side effects (6). Many studies evaluated this combination with different treatment schedules and they showed response rates varying between 37 and $42 \%$ and a median overall survival (OS) period of between 8.4 and 13.5 months (6-11). A recent meta-analysis showed an absolute benefit (3.9\%) in 1-year OS in favor of regimens containing gemcitabine and platinum, with a significant reduction in both overall mortality and disease progression (12). Thus, gemcitabine combined with cisplatin is currently considered to be one of the standard regimens in the treatment of advanced NSCLC (13-15).

Docetaxel, a semisynthetic taxoid, possesses significant activity as a single agent in the treatment of patients with NSCLC. A review demonstrated that the results from significant phase I and II studies showed significant antitumor activity and tolerability of docetaxel combined with platinum compounds for patients with advanced NSCLC (16). Thus, the effectiveness of docetaxel as a single agent or cisplatin-based doublet has already been accepted in treatment of advanced NSCLC $(15,17,18)$.

The aim of this study was to investigate the feasibility and effectiveness of cisplatin-gemcitabine-docetaxel (CGD) triplet given weekly in patients with chemotherapy-naive advanced stage NSCLC.

\section{Materials and methods}

Patient eligibility criteria. Histologically or cytologically confirmed NSCLC patients with Eastern Cooperative 
Oncology Group (ECOG) performance status (PS) of 0 to 2 and advanced stage disease (IIIB/IV) were included. Patients who had brain metastases were required to have completed cranial radiotherapy at least 3 weeks prior to triplet chemotherapy with a stable neurologic status. Other inclusion criteria were adequate functioning of bone marrow (white blood cell count $\geq 3,000 / \mathrm{mm}^{3}$, hemoglobin $\geq 9 \mathrm{~g} / \mathrm{dl}$, platelets $\geq 100,000 / \mathrm{mm}^{3}$ ), liver (total bilirubin level $\leq 1.5 \mathrm{mg} / \mathrm{dl}$, alanine and aspartate transaminase levels less than twice the upper limit of normal) and kidney (serum creatinine level $<1.5 \mathrm{mg} / \mathrm{dl}$ ). Patients with a previous history of chemotherapy for advanced stage NSCLC, and major comorbidities such as severe cardiopulmonary dysfunction, recent history of myocardial ischemia, uncontrolled arrhythmia and active infection were excluded. All patients gave written informed consent before treatment and the study was approved by the local institutional scientific committee.

Baseline evaluation. Pretreatment assessment routinely included medical history, physical examination, complete blood count, serum biochemistry, electrocardiogram (ECG), chest computed tomography (CT), abdominal CT and/or magnetic resonance imaging (MRI), bone scan or positron emission tomography (PET/CT) and cranial MRI when indicated. Tumor stages were assessed according to the TNM classification of International Union Against Cancer (19).

Treatment schedule. Gemcitabine was administered intravenously (i.v.) as a $30 \mathrm{~min}$ infusion with saline at a dose of $750 \mathrm{mg} / \mathrm{m}^{2}$, cisplatin and docetaxel were given i.v. as a $1 \mathrm{~h}$ infusion with saline at a dose of $25 \mathrm{mg} / \mathrm{m}^{2}$ on days 1,8 , and 15 . Treatment cycles were repeated every 3 weeks on an outpatient basis unless disease progression or severe toxicity was experienced. Premedication consisted of antiemetic combination with standard serotonin antagonists and dexamethasone. Granulocyte colony stimulating factor (G-CSF) was not used prophylactically during the first cycle of the study, but it was recommended for patients who had previously experienced either febrile neutropenia or grade IV neutropenia lasting more than 5 days. All patients who developed febrile neutropenia were also eligible for prophylactic growth factor administration in the next cycles.

Toxicity and dose delay or modifications. Complete blood counts were performed on a weekly basis. Serum biochemistry and physical examination including the determination of ECOG PS and vital signs were performed one day before each chemotherapy cycle. Common toxicity criteria of $\mathrm{NCI}$ (CTCv3.0) had been used. The doses of chemotherapeutic agents were modified for hematological and severe nonhematological toxicities except for emesis. Treatment was delayed for one week if there was not full hematological recovery from the prior cycle of treatment. Patients requiring more than two dose reductions or a delay of more than 3 weeks were removed from the study. Patients with progressive disease at any time were withdrawn from the study.

Response evaluation. Before each cycle, common toxicity criteria, PS and measurement of clinically assessable disease were documented. Patients were evaluated for response if
Table I. Characteristics of patients and tumors.

\begin{tabular}{lc}
\hline Parameter & Value \\
\hline Age (years) & \\
Median & 59 \\
Range & $38-72$ \\
Gender, $n$ & \\
Female & 1 \\
Male & 26 \\
Performance status, $\mathrm{n}$ & \\
0 & 14 \\
1 & 9 \\
2 & 4 \\
Histology, $\mathrm{n}$ & \\
Adenocarcinoma & 13 \\
Squamous cell carcinoma & 11 \\
Undifferentiated & 3 \\
Tumor stage, $\mathrm{n}$ & \\
IIIB & 2 \\
IV & 25 \\
\hline
\end{tabular}

they received one or more cycles of treatment. If there was no sign or symptom of progression after one cycle, response was assessed at the end of second chemotherapy cycle. Tumor response was evaluated by physical examination, imaging radiograms such as CT, MR, US and/or PET/CT. Patients with stable disease or partial response after the second cycle continued to a maximum of 4 cycles unless intolerance developed. Two additional cycles of chemotherapy were given to responsive patients. Patients with progression or severe toxicity after sufficient dose reduction discontinued the treatment.

Statistical analyses. SPSS software (SPSS version 16, Chicago, IL, USA) was used for statistical analyses. Quantitative analyses were summarized by mean, standard error, median, minimum and maximum, and qualitative analyses were presented as frequencies and percentages. Survival analyses were estimated by the Kaplan-Meier method. $\mathrm{P} \leq 0.05$ was considered to indicate a statistically significant result. The overall response rate in previous studies with the same triplet GCD showed a success rate of $34 \%$ (20). New patients were included in the study until a $95 \%$ confidence interval rate was reached. New patient admission was stopped after the first patient had a one year follow-up. Sample size was determined using Simon's tables.

OS was determined as the time elapsed between the time of histological diagnosis and the date of mortality or the date of the last follow-up visit or the date of the study written, if the patient was still alive at this time. Progression-free survival (PFS) was recorded from the day of histological diagnosis to the date of first documented progressive disease or the date of mortality, regardless of its course, or to date of point if no progressive disease and no mortality appeared at this time. 
Table II. Toxicity profile.

\begin{tabular}{|c|c|c|c|c|}
\hline \multirow[b]{2}{*}{ Toxicity } & \multicolumn{4}{|c|}{ NCI toxicity (no. of patients) } \\
\hline & I & II & III & IV \\
\hline \multicolumn{5}{|l|}{ Hematological } \\
\hline Leucopenia & 1 & 10 & 5 & 0 \\
\hline Neutropenia & 2 & 7 & 6 & 1 \\
\hline Anemia & 3 & 14 & 0 & 0 \\
\hline Thrombocytopenia & 3 & 4 & 2 & 0 \\
\hline \multicolumn{5}{|l|}{ Biochemical } \\
\hline Creatinine & 2 & 0 & 0 & 0 \\
\hline Transaminase & 0 & 2 & 0 & 0 \\
\hline ALP & 0 & 0 & 1 & 0 \\
\hline Bilirubin & 0 & 0 & 0 & 0 \\
\hline \multicolumn{5}{|l|}{ Non-hematological } \\
\hline Alopecia & 5 & 5 & 0 & 0 \\
\hline Diarrhea & 5 & 0 & 1 & 0 \\
\hline Constipation & 3 & 0 & 0 & 0 \\
\hline Nausea & 10 & 5 & 0 & 0 \\
\hline Vomiting & 5 & 6 & 0 & 0 \\
\hline Pulmonary & 2 & 4 & 1 & 2 \\
\hline Stomatitis & 7 & 2 & 0 & 0 \\
\hline Fatigue & 1 & 1 & 0 & 1 \\
\hline Insomnia & 0 & 2 & 0 & 0 \\
\hline Allergic reaction & 2 & 0 & 0 & 0 \\
\hline Dysphasia & 1 & 1 & 0 & 0 \\
\hline Hearing loss & 0 & 0 & 0 & 0 \\
\hline
\end{tabular}

ALP, alkaline phosphatase; NCI, National Cancer Institute.

\section{Results}

Patients. In total, 27 patients (26 males and 1 female) were treated with triplet CGD regimen between January 2008 and December 2009. The clinical characteristics of the patients are listed in Table I. The median age of patients was 59 years (range, 38-72 years). The majority were male and had PS 0-1 and stage IV disease.

Safety. The toxicity profiles of all 27 patients are shown in Table II. Hematological toxicity was found to be the principal dose-limiting toxicity. Severe, grade III/IV neutropenia and leucopenia were observed in 26 and $19 \%$ of the patients, respectively. One patient developed febrile neutropenia with an underlying empyema and pneumothorax. Two patients required G-CSF usage due to febrile neutropenia and grade IV neutropenia without fever. Only 2 patients (7\%) developed grade III thrombocytopenia. None of the patients had grade III/IV anemia. However, due to symptomatic grade II anemia, 7 patients (26\%) received a total of 12 units of packed red blood cells.

Patients were treated with various antiemetics containing seratonine antagonists. Several other severe non-hematological
Table III. Response evaluation.

\begin{tabular}{lrrr}
\hline Response & No. & $\%$ & $95 \%$ CI \\
\hline Complete response & 0 & 0 & $0-13$ \\
Partial response & 14 & 52 & $32-71$ \\
Stable disease & 2 & 7 & $1-24$ \\
Progression & 11 & 41 & $22-61$ \\
\hline CI, confidence interval. & & & \\
\hline
\end{tabular}

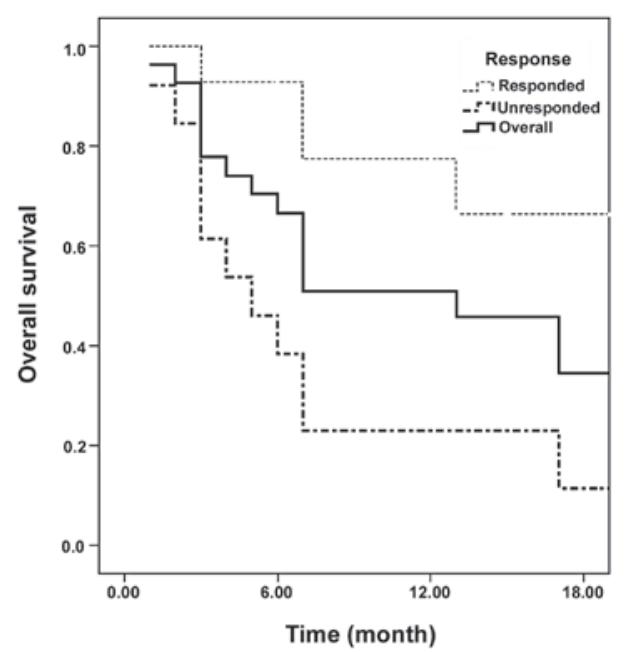

Figure 1. Overall survival (OS) curves.

side effects were managed according to the standard protocols. No toxic or early mortality was observed.

Dose delivery. The patients received a total of 85 cycles of CGD with a median of 4 cycles per patient (range, 1-6). Permanent $25 \%$ dose reductions were necessary in 7 (26\%) patients due to severe hematological and non-hematological toxicity. Chemotherapy administration was also delayed in $12(44 \%)$ patients due to delayed hematological or non-hematological toxicity recovery. Treatment was discontinued in $4(15 \%)$ patients due to severe fatigue and deteriorating performance status ( 2 patients) or disease progression ( 2 patients) after 1-3 cycles of chemotherapy.

Efficacy. All 27 patients were evaluated for response (Table III). The overall response rate for all patients was 52\% and all of them had partial response. Two patients (7\%) had stable disease and the remaining 11 patients (41\%) showed progression under treatment.

Survival analyses. The median follow-up of the patients was 7 months (range, 2-18). At the time of last follow-up, $10(37 \%)$ patients were alive. The median PFS was 6 months (95\% CI, 3-9). The median OS was 13 months (95\% CI, 6-20) (Fig. 1). The 1-year OS rate was $46 \%$ (range, $2-30$ months). The median PFS of responders to chemotherapy was 11 months (95\% CI, 9-13) and the median PFS of non-responders was 
3 months (95\% CI, 1-5) (P=0.02). The median OS of responders to chemotherapy could not be reached, as sufficient mortality was not observed; however, the median OS of non-responders was 5 months (95\% CI, 2-9) ( $\mathrm{P}=0.01$; Fig. 1).

\section{Discussion}

Based on the results of several randomized trials, there is sufficient evidence to conclude that adding a drug to a single agent or to a two-agent regimen increased the tumor response rate in patients with advanced NSCLC, although its impact on survival remains controversial $(21,22)$.

A recent meta-analysis showed that adding an agent to doublet therapy did not provide a better survival than that of doublet chemotherapy (23). Additionally, toxicity was increased significantly. Most of the studies, including that meta-analysis, combined first or second generation agents. It was suggested that the third generation agent was diluted and had less effect. A review of the studies performed with triplet cytotoxic chemotherapy in advanced NSCLC demonstrated that triplet therapy with third generation agents increased response rates of tumors at the expense of increased toxicity (24). Although triplet chemotherapy had a better OS compared with doublet therapy, it was not statistically significant.

Phase I trials determined that $75 \mathrm{mg} / \mathrm{m}^{2}$ docetaxel and $75 \mathrm{mg} / \mathrm{m}^{2}$ cisplatin is the recommended dose for phase II and III trials (16). Overall, response rates with docetaxel and cisplatin have ranged from 21 to $48 \%$. Median survival of 8 to 13 months has been achieved in phase II trials. A recent phase I/II trial investigated weekly consecutive administration of docetaxel at a dose of $40 \mathrm{mg} / \mathrm{m}^{2}$ on days 1,8 and 15 for 3 weeks plus cisplatin at a dose of $75 \mathrm{mg} / \mathrm{m}^{2}$ on day 1 every 4 weeks (25). It was found to be tolerable and effective (objective response rate of $27.7 \%$ ) with minimal myelosuppression in chemotherapy-naive patients with advanced NSCLC (26).

A phase I/II study with cisplatin, gemcitabine and docetaxel in patients with advanced NSCLC has been conducted (20). All drugs (cisplatin $40 \mathrm{mg} / \mathrm{m}^{2}$, docetaxel $30 \mathrm{mg} / \mathrm{m}^{2}$ and gemcitabine $800 \mathrm{mg} / \mathrm{m}^{2}$ ) were given on days 1 and 8 , repeated every 3 weeks. The objective response rate was found to be $34 \%$ with tolerable toxicity. As the possibility of obtaining better response and survival gain, but same levels of toxicity was shown when comparing with cisplatin-based doublets, we conducted the current study with this triplet combination of CGD with weekly schema in order to lower side effects.

This is the first clinical study of scheduled weekly continuous administration evaluating the efficacy of this combination in this setting. The current study demonstrated that the combination of two new generation drugs, gemcitabine and docetaxel, with cisplatin and weekly continuous administration of this triplet, has favorable antitumor activity in patients with chemotherapy-naive advanced NSCLC. When compared with cisplatin-based doublet regimens with either gemcitabine or docetaxel, concurrent delivery of both drugs with cisplatin weekly appears to enhance antineoplastic activity, as shown by $\sim 50 \%$ higher response rate in this study than in those previously reported (20).

This weekly triplet regimen in the present study was also found to be safe and well tolerated and adverse events were mild to moderate in the majority of the patients. Compared with regimens with one week interruption, weekly continuous administration of triplet chemotherapy regimen diminishes the frequency of myelotoxicity, nausea, infection (which were the principle toxicities) or other adverse events. Myelosuppression was infrequent and readily manageable. Only one patient had febrile neutropenia and myelosuppression-related discontinuation of treatment did not occur in any patient. Moreover, there were no treatment-related mortalities. The dose and schedule of treatment used in the current study appears to be tolerable and reasonable for patients in this setting.

In conclusion, cisplatin-based triplet treatment with new generation drugs increased the response rate with acceptable side effects and improved OS or PFS. This should encourage further studies with triplet cytotoxic chemotherapy regimens at reduced doses as first line chemotherapy in advanced NSCLC patients.

\section{References}

1. Marino P, Pampallona S, Preatoni A, Cantoni A, Invernizzi F: Chemotherapy vs supportive care in advanced non-small-cell lung cancer. Results of a meta-analysis of the literature. Chest 106: 861-865, 1994.

2. No authors listed. Chemotherapy in non-small cell lung cancer: a meta-analysis using updated data on individual patients from 52 randomized clinical trials. Non-small Cell Lung Cancer Collaborative Group. BMJ 311: 899-909, 1995.

3. Bonomi P, Kim K, Fairclough D, Cella D, Kugler J, Rowinsky E, et al: Comparison of survival and quality of life in advanced non-small-cell lung cancer patients treated with two dose levels of paclitaxel combined with cisplatin versus etoposide with cisplatin: results of an Eastern Cooperative Oncology Group trial. J Clin Oncol 18: 623-631, 2000.

4. Le Chevalier T, Brisgand D, Douillard JY, Pujol JL, Alberola V, Monnier A, et al: Randomized study of vinorelbine and cisplatin versus vindesine and cisplatin versus vinorelbine alone in advanced non-small-cell lung cancer: results of a European multicenter trial including 612 patients. J Clin Oncol 12: 360-367, 1994.

5. Metro G, Cappuzzo F, Finocchiaro G, Toschi L and Crinò L: Development of gemcitabine in non-small cell lung cancer: the Italian contribution. Ann Oncol 17: 37-46, 2006.

6. Bergman AM, Ruiz van Haperen VW, Veerman G, Kuiper CM and Peters GJ: Synergistic interaction between cisplatin and gemcitabine in vitro. Clin Cancer Res 2: 521-530, 1996.

7. Lippe P, Tummarello D, Monterubbianesi MC, Silva RR, Giuliodori L, Mari D, et al: Weekly gemcitabine and cisplatin in advanced non-small-cell lung cancer: a phase II study. Ann Oncol 10: 217-221, 1999.

8. Abratt RP, Bezwoda WR, Goedhals L and Hacking DJ: Weekly gemcitabine with monthly cisplatin: effective chemotherapy for advanced non-small-cell lung cancer. J Clin Oncol 15: 744-749, 1997.

9. Einhorn LH: Phase II trial of gemcitabine plus cisplatin in non-small cell lung cancer: a Hoosier Oncology Group study. Semin Oncol 24: S824-S826, 1997.

10. Crinò L, Scagliotti G, Marangolo M, Figoli F, Clerici M, De Marinis F, et al: Cisplatin-gemcitabine combination in advanced non-small-cell lung cancer: a phase II study. J Clin Oncol 15: 297-303, 1997.

11. Chen SC, Lin MC, Chang JW, Wang SW, Lee CH and Tsao TC: Phase II study of regimen of gemcitabine and cisplatin in advanced non-small cell lung cancer. Jpn J Clin Oncol 30: 494-498, 2000

12. Le Chevalier T, Scagliotti G, Natale R, Danson S, Rosell R, Stahel R, et al: Efficacy of gemcitabine plus platinum chemotherapy compared with other platinum containing regimens in advanced non-small-cell lung cancer: a meta-analysis of survival outcomes. Lung Cancer 47: 69-80, 2005.

13. Crinò L, Scagliotti GV, Ricci S, De Marinis F, Rinaldi M, Gridelli C, et al: Gemcitabine and cisplatin versus mitomycin, ifosfamide, and cisplatin in advanced non-small-cell lung cancer: A randomized phase III study of the Italian Lung Cancer Project. J Clin Oncol 17: 3522-3530, 1999. 
14. Sandler AB, Nemunaitis J, Denham C, von Pawel J, Cormier Y, Gatzemeier U, et al: Phase III trial of gemcitabine plus cisplatin versus cisplatin alone in patients with locally advanced or metastatic non-small-cell lung cancer. J Clin Oncol 18: 122-130, 2000.

15. Schiller JH, Harrington D, Belani CP, Langer C, Sandler A, Krook $\mathrm{J}$, et al: Comparison of four chemotherapy regimens for advanced non-small-cell lung cancer. N Engl J Med 346: 92-98, 2002.

16. Belani CP: Docetaxel in combination with platinums in patients with advanced non-small-cell lung cancer. Oncology 11: 42-45, 1997.

17. Fossella F, Pereira JR, von Pawel J, Pluzanska A, Gorbounova V, Kaukel E, et al: Randomized, multinational, phase III study of docetaxel plus platinum combinations versus vinorelbine plus cisplatin for advanced non-small-cell lung cancer: the TAX 326 study group. J Clin Oncol 21: 3016-3024, 2003.

18. Kubota K, Watanabe K, Kunitoh H, Noda K, Ichinose Y, Katakami N, et al: Phase III randomized trial of docetaxel plus cisplatin versus vindesine plus cisplatin in patients with stage IV non-small-cell lung cancer: the Japanese Taxotere Lung Cancer Study Group. J Clin Oncol 22: 254-261, 2004.

19. Sobin LH and Fleming ID: TNM Classification of Malignant Tumors, fifth edition (1997). Union Internationale Contre le Cancer and the American Joint Committee on Cancer. Cancer 80: 1803-1804, 1997.

20. Tabata M, Kozuki T, Ueoka H, Nicolella GP, Natale M, Pacilio C, et al: A triplet chemotherapy with cisplatin, docetaxel and gemcitabine in patients with advanced non-small-cell lung cancer: a phase I/II study. Cancer Chemother Pharmacol 60: 53-59, 2007.
21. Frasci G, Panza N, Comella P, Manzione L, De Cataldis G, Cioffi R, et al: Cisplatin, gemcitabine and vinorelbine in locally advanced or metastatic non-small-cell lung cancer: a phase I study. Ann Oncol 8: 1045-1048, 1997.

22. Comella P, Frasci G, Panza N, Soria JC, Le Chevalier T and Pignon P: Randomized trial comparing cisplatin, gemcitabine, and vinorelbine with either cisplatin and gemcitabine or cisplatin and vinorelbine in advanced non-small-cell lung cancer: interim analysis of a phase III trial of the Southern Italy Cooperative Oncology Group. J Clin Oncol 18: 1451-1457, 2000.

23. Delbaldo C, Michiels S, Syz N, Soria JC, Le Chevalier T and Pignon JP: Benefits of adding a drug to a single-agent or a 2 -agent chemotherapy regimen in advanced non-small-cell lung cancer: a meta-analysis. JAMA 292: 470-484, 2004.

24. Azim HA Jr, Elattar I, Loberiza FR Jr, Azim H, Mok T and Ganti AK: Third generation triplet cytotoxic chemotherapy in advanced non-small cell lung cancer: a systematic overview. Lung Cancer 64: 194-198, 2009.

25. Li JL, Zhang XR, Liu JW, Fujiwara Y, Toyooka S and Umemura S: Phase I/II clinical trial of weekly administration of docetaxel plus cisplatin for advanced non-small cell lung cancer.Zhonghua Zhong Liu Za Zhi 28: 309-312, 2006 (In Chinese).

26. Fujimoto N, Kiura K, Takigawa N, Kiura K, Harita S, Tada A, et al: Triplet chemotherapy with cisplatin, docetaxel, and irinotecan for patients with recurrent or refractory non-small cell lung cancer. Acta Med Okayama 64: 33-37, 2010. 